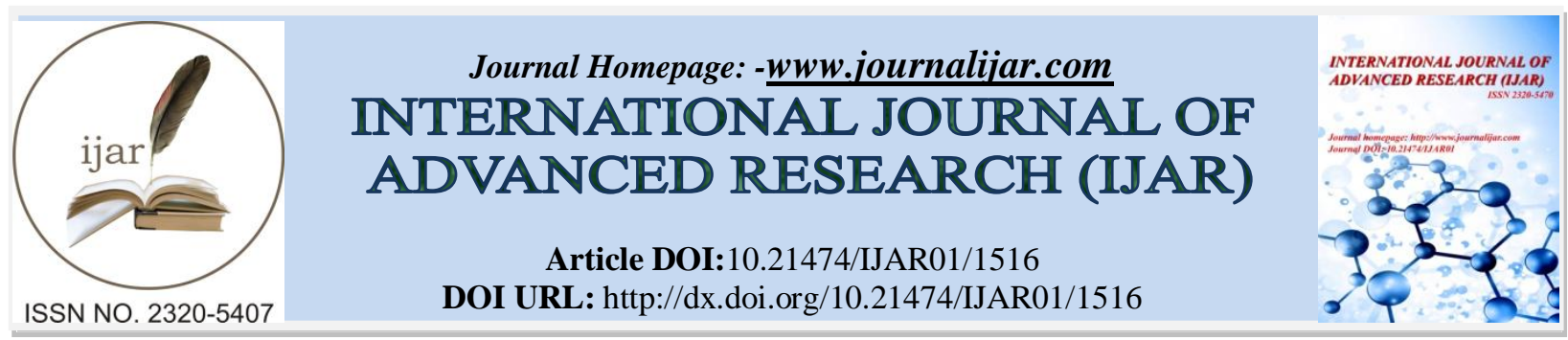

RESEARCH ARTICLE

\title{
MULTIPLE ACTIVE SPATIAL MODULATION IN MIMO SYSTEMS
}

\author{
Nicy Anto. \\ MTech Student, Dept. of ECE, Rajagiri School of Engineering and Technology, Kakkanad,Cochin,Kerala,India.
}

\section{Manuscript Info}

Manuscript History

Received: 12 July 2016

Final Accepted: 11 August 2016

Published: September 2016

Key words:-

Multiple Active Spatial Modulation (MASM), Vertical Bell Lab Layered Space Time (VBLAST), Generalized Spatial Modulation (GSM), Spatial modulation (SM), Space Time Block Code (STBC), Maximum Likelihood (ML).

\section{Abstract}

A generalised spatial modulation scheme with multiple active transmit antennas known as multiple active spatial modulation is introduced in this paper. MA-SM has advantages of the low complexity and high multiplexing gain. The transmitted symbols are mapped into a higher dimensional constellation space which includes the spatial dimension. The general principle for the design of MA-SM for arbitrary number of transmit antennas and modulation scheme is described. The detection scheme is near optimal and less complex. A comparison with the existing multiple-input multiple output (MIMO) techniques such as space time block code (STBC) and V-BLAST is also presented which shows the efficiency of MA-SM

Copy Right, IJAR, 2016,. All rights reserved.

\section{Introduction:-}

Multiple input multiple-output (MIMO) is an effective way to improve capacity and reliability compared to single antenna wireless systems. Out of the several MIMO techniques space time block code (STBC) and spatial multiplexing are the techniques achieving diversity and multiplexing gain, respectively. STBC provide excellent spatial diversity gain and also there is simplicity in implementation and also it has low decoding complexity. The orthogonal STBC (OSTBC) which is a classification of STBC uses maximum likelihood (ML) decoder with linear complexity. However, it is not possible to construct a full-rate full-diversity code with linear complexity.

V-BLAST is the family of spatial multiplexing, which can provide high multiplexing gain. V-BLAST can achieve maximum multiplexing gain by simultaneous transmission on all the antennas. But Inter Channel Interference (ICI) and Inter Antenna Interference (IAI) make it difficult to decode streams. The capacity gain can be improved by the introduction of spatial dimension and it is coordinated by the antenna indices.

Jeganathan[2] introduced space shift keying (SSK) modulation scheme in which amplitude/phase modulation is removed and only antenna indices are used to convey information. Since only one antenna is active during a time slot, the ICI and IAI can be avoided fully. So system design is simplified and the decoding complexity reduced. Higher capacity could be achieved by combining the amplitude/phase modulation (APM) with antenna index modulation, known as Spatial Modulation (SM) It extend the constellation into a three dimension (both the complex dimension and the spatial dimension)Symbols are ejected from a selected antenna after mapped through a traditional modulator. Therefore, the information is transmitted by amplitude/phase modulation and also by the antenna indices. The Inter Channel Interference and Inter antenna interference can be avoided since only one antenna is active over the transmission. The decoder complexity also gets reduced. 
In SM and SSK modulation systems only one antenna is active which limits the exploitation of spatial dimension and design flexibility. An extension of SM, named as generalised spatial modulation (GSM) allows several antennas to be active simultaneously. It exploits high spectral efficiency. But it increases the complexity of the system exponentially. Moreover, efficient decoders for GSM are highly complex while corresponding linear decoder has poor performance.

In Space Time Block Coding-Spatial Modulation (STBC-SM) scheme, SM is combined with Space Time Block Code (STBC). At the transmitter, mapped symbols are emitted from chosen antennas after coded with STBC encoder. At the receiver, a demodulator combining ML algorithm with the linear STBC decoder is used which is optimal. STBC-SM has the problem of low multiplexing gain or high computational complexity if the block code size is greater than two. An exhaustive search of antenna sets is employed in ML decoder for STBC-SM, and thus the complexity increases exponentially as the antenna subset expands. In order to explore the multiplexing gain of system with low computational complexity, a novel multiple active spatial modulation (MASM) scheme and a nearoptimal decoder with linear complexity is introduced.

A novel scheme of multi-antenna transmission for SM is proposed in which several transmit antennas carrying different information symbols are active during each time slot which is called Multiple Active Spatial Modulation. As in traditional spatial modulation, information bits are mapped into both spatial dimension and traditional complex dimension. Antenna sets with arbitrary number of active antennas is used instead of using a single antenna index to improve the multiplexing gains. A near-optimal decoder withlow complexity is proposed .Thedecoder separates the antenna set detection from the traditional demodulator which reduces the computational complexity. Compared to the Maximum likelihood detection the proposed method is less complex computationally.

\section{Proposed MASM System:-}

The system model consists of a MIMO wireless link with $N_{T}$ transmit antennas and $N_{R}$ receive antennas. The source information bits are transmitted from $N_{P}$ of the transmit antennas after mapped through an $M$-QAM. Through the $N_{T} \times N_{R}$ wireless channel $H$ and the $N_{R}$ dimension additive white Gaussian noise (AWGN) $w=\left[w_{1} w_{2} \ldots \ldots w_{N_{R}}\right]$, the received signal is given by (1) where $\rho$ is the average signal to noise ratio (SNR) at each receive antenna, $H$ and $w$ are independent and identically distributed (i.i.d) entries according to $\mathcal{C N}(0,1)$ (complex Gaussian zero mean distribution with variance unity) and $\mathcal{S}$ is the constellation set of M-QAM.

The transmitted symbol $X$ is comprised with $N_{P}$ QAM symbols emitted from the antennas $a_{1} \ldots a_{N_{P}}$, respectively. For denoting convenience, the antenna group $\left(a_{1} \ldots a_{P}\right)$ will be written as $\xi_{j}=$ $\left.(0,1, \ldots . .1 \ldots .). j \epsilon\left\{1, \ldots \ldots . \mid\left(\begin{array}{l}N_{T} \\ N_{P}\end{array}\right)\right]_{2}\right\}$ where the $N_{T}$ components stands for the states of $N_{T}$ transmit antennas and each 1 and 0 represents the on and off of the corresponding antenna, respectively. For example, in a system where $N_{T}=4, N_{P}=2$, the possible antenna groups could be denoted $\operatorname{as} \xi_{1}=(1,1,0,0), \xi_{2}(1,0,1,0), \xi_{3}(1,0,0,1), \xi_{4}(0,1,1,0), \xi_{5}=(0,1,0,1), \xi_{6}=(0,0,1,1)$

$$
\begin{aligned}
& y=\sqrt{\frac{\rho}{N_{P}}} H X+w \\
& X \triangleq\left[\begin{array}{llll}
0 & s_{1} \ldots s_{N_{P}} & 0
\end{array}\right]^{T} \\
& \uparrow \uparrow \\
& a_{1} a_{N_{P}} \\
& \text { where } s_{1} \ldots \ldots \ldots s_{N_{P}} \in \mathcal{S}, a_{1} \ldots \ldots \ldots \ldots a_{N_{P}} \in\left\{1 \ldots \ldots . N_{P}\right\} \text {. }
\end{aligned}
$$

This equation could be simplified to (2) where $h_{a_{i}}$ denote $a_{i}{ }^{\text {th }}$

Column of channel matrix $H$

$$
y=\sqrt{\frac{\rho}{N_{P}}}\left[h_{a_{1}} \ldots \ldots \ldots h_{a_{N_{P}}}\right]\left[s_{1} \ldots \ldots \ldots s_{N_{p}}\right]^{T}+w(2)
$$


If $N_{P}$ from $N_{T}$ antennas are selected $\left[\log _{2}\left(\begin{array}{l}N_{T} \\ N_{P}\end{array}\right)\right]$ bits can be conveyed on antenna indices. At high signal to noise ratio the capacity of MASM is $N_{P} C_{\text {norm }}+\left[\log _{2}\left(\begin{array}{l}N_{T} \\ N_{P}\end{array}\right)\right]$ where $C_{\text {norm }}=\log _{2}\left(1+\frac{S N R}{N_{P}}\right)$ is the capacity of a single channel in MIMO system

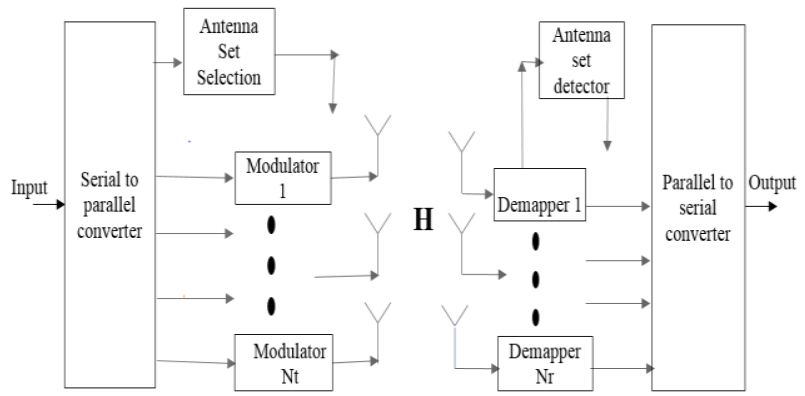

Fig 1:- System Model

The block diagram of the MASM system is given in figure 1 which shows $N_{P}$ antennas from $N_{T}$ are selected as the antenna In the MA-SM system, the information bits are conveyed by both the complex symbols and the indices of the active antennas. At the transmitter, $N_{P}$ antennas are used to carry different symbols, which increases the multiplexing gain. $N_{P}$ is taken as a tradeoff between capacity and reliance. Active antenna set has to be selected carefully so that interference can be minimized. Transmission scheme can be optimized by minimizing the maximum distance involved.

The optimal ML decoder detects the antenna set together with symbols and thus complexity increases exponentially for higher order constellation. Thus a near optimal decoder with low complexity is proposed. The decoder can be interpreted as the linear filter that maximizes the output SNR under the constraint that it eliminates the interference coming from all other data streams.

\section{Complexity and Analysis:-}

Assume a $4 \times 4$ MIMO system with $N_{P}=2$ and 16QAM modulation scheme, the optimal ML needs 16384 multiplications while the proposed decoder only employs 43 multiplications. As the number of multiplications is less the proposed method is less computationally complex. Also the complexity of the proposed scheme is unrelated to the size of QAM employed.

The error that occur in the demodulator could be categorized into two categories. The first is the error occurs in the active antenna detection $\left(P_{\text {AntErr }}\right)$ and the second is that the error occurs in conventional demapping when antenna detection is correct $\left(P_{\text {ModErr }}\right)$.

\section{Simulation Results:-}

Simulation results for the MA-SM system with comparisons among other MIMO systems, such as STBC, V-BLAST are given. The bit error rate (BER) performance of these systems as a function of the average SNR per receive antenna and in all cases is plotted.

Figure 2,3 and 4 shows the performance of MASM for BPSK,QPSK and 16QAM modulation techniques compared to STBC and VBLAST. It clearly shows that in all the casesFigure 5 shows the comparison of MA-SM with different modulation schemes. It shows that after $10 \mathrm{~dB}$ SNR MA-SM shows better performance.Also on comparison with different modulation schemes 16-QAM has better result. 


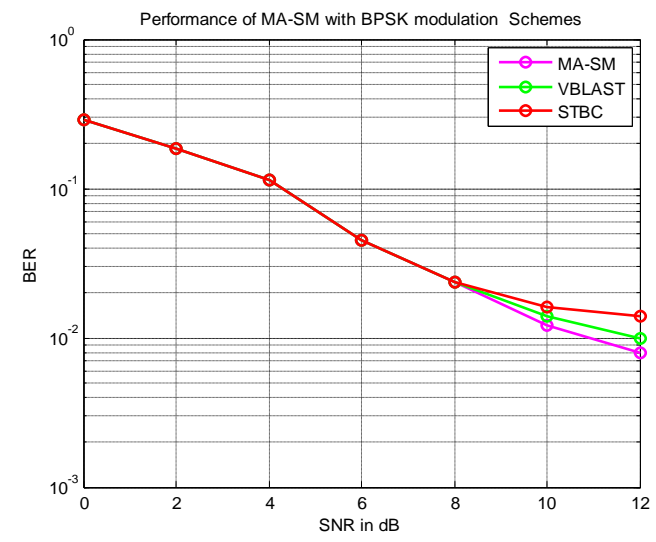

Fig 2:- Comparison of MASM with existing techniques for BPSK modulation.

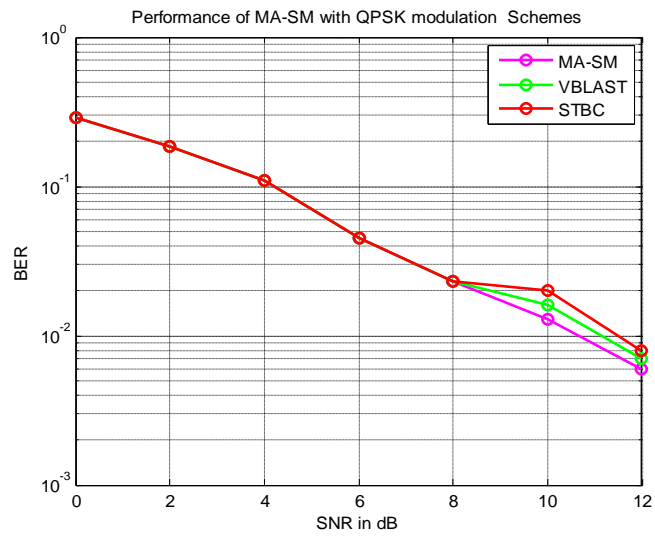

Fig 3:- Comparison of MASM with existing techniques for QPSK modulation.

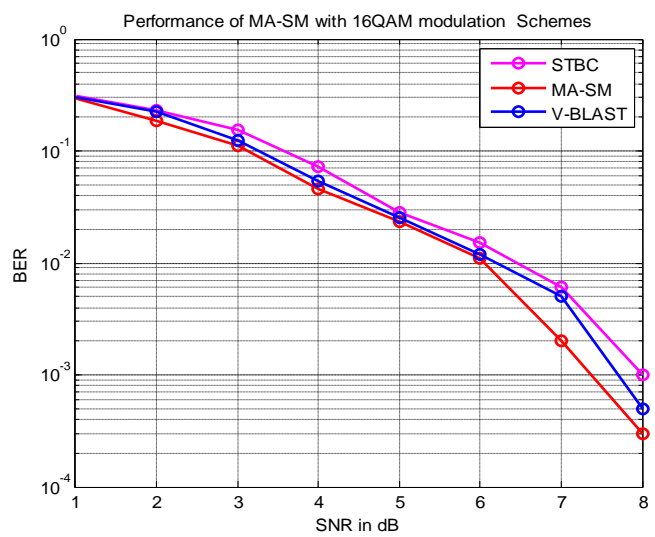

Fig 4:- Comparison of MASM with existing techniques for 16QAM modulation. 


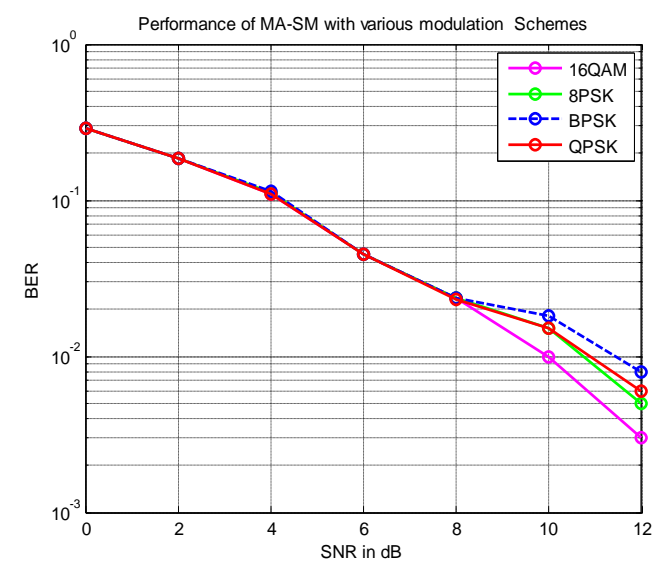

Fig 5:- MASM with different modulation schemes

\section{Conclusions:-}

In this paper, a novel high-rate, low complexity MIMO scheme, namely MASM, as an alternative to existing techniques like STBC and VBLAST is proposed. The proposed MASM scheme employs both traditional modulation and antenna indices to convey information. MA-SM offers significant improvements of system performance compared to VBLAST and STBC systems with linear decoding complexity. It can be concluded that the MASM scheme can be useful for high data rate communication systems

\section{References:-}

1. S. M. Alamouti, "A simple transmit diversity technique for wireless communications," IEEE J. Sel. Areas Commun., vol. 16, pp. 1451-1458, Oct. 1988.

2. J. Jeganathan, A. Ghrayeb, and L. Szczecinski, "Spatial modulation: optimal detection and performance analysis," IEEE Trans. Commun Lett., vol. 12, pp. 545-547, Aug. 2008

3. R. Mesleh, H. Haas, S. Sinaovic, C. W. Ahn, and S. Yun, "Spatial modulation," IEEE Trans. Veh. Technol., vol. 57, no. 4, pp. 2228-2241, July 2008R. Nicole, "Title of paper with only first word capitalized," J. Name Stand. Abbrev., in press.

4. Jintao Wang, Shuyun Jia, and Jian Song "Generalised Spatial Modulation System with Multiple Active Transmit Antennas and Low Complexity Detection Scheme", IEEE transactions on wireless communications, Vol. 11, No. 4, April 2012

5. Karthika V. Nair, Poorna R. Prabhu "A Comparative Study on Multiple Active Spatial Modulation in MIMO Systems",International Conference on Control, Instrumentation, Communication and Computational Technologies (ICCICCT), 2014

6. P. Wolniansky, G. Foschini, G. Golden, and R. Valenzuela, "Vblast: an architecture for realizing very high data rates over the rich-scattering wireless channel", in Proc. 1998 International Symp. Signals, Syst., $\quad$ Electron., pp. 295-300.

7. A. Younis, N. Serafimovski, R. Mesleh, and H. Haas, "Generalised spatial modulation", in Proc. 2010 Signals, Syst. Comput., pp. 1498-1502

8. J. Fu, C. Hou, W. Xiang, L. Yan, and Y. Hou, "Generalistedspatialmodulation with multiple active transmit antennas", in Proc. 2010 IEEE Globecom Workshops, pp. 839-844 